J. Dairy Sci. 95:5569-5579

http://dx.doi.org/10.3168/jds.2012-5501

(C) American Dairy Science Association ${ }^{\circledR}, 2012$.

\title{
Steady shear rheological properties of micellar casein concentrates obtained by membrane filtration as a function of shear rate, concentration, and temperature
}

\author{
A. Sauer, ${ }^{*}$ I. Doehner, † and C. I. Moraru*1 \\ *Department of Food Science, Cornell University, Ithaca, NY 14853 \\ †Technical University of Berlin, Berlin, Germany
}

\begin{abstract}
The use of casein preparations obtained by membrane separation is receiving increasing interest from the dairy and food industry. The objective of this work was to generate information about the steady shear rheological properties of micellar casein concentrates (MCC) and the effect of composition, temperature, and shear rate on these properties. Micellar casein concentrate preparations with 2 levels of serum proteins (SP; 65 and $95 \%$ SP reduced, respectively), were obtained from skim milk by microfiltration followed by spray drying. Micellar casein concentrate preparations with casein concentrations ranging from 2.5 to $12.5 \%$ were obtained by dispersing the MCC powders in ultrapure water. Steady shear rheological analyses at temperatures ranging from 0 to $80^{\circ} \mathrm{C}$ were performed using a strain-controlled rheometer. Viscosity versus shear rate curves were used to evaluate the effect of shear on viscosity, and the apparent viscosity at a shear rate of $100 \mathrm{~s}^{-1}$ was used to make direct comparisons between various concentration and temperature conditions. The $65 \%$ SP-reduced MCC had lower viscosity than the $95 \%$ SP-reduced MCC at the same casein concentration and temperature. Protein preparations at casein concentrations above $7.5 \%$ displayed shear-thinning behavior, which was more pronounced as concentration increased. The viscosity of MCC increased exponentially with casein concentration and decreased with temperature. The dependency of viscosity on temperature followed an Arrhenius relationship. A modified Arrhenius model able to accurately predict rheological properties under desired shear, temperature, and concentration conditions was developed and validated. This study provides critical rheological data necessary for developing practical applications of micellar casein preparations.
\end{abstract}

\footnotetext{
Received March 3, 2012.

Accepted June 22, 2012.

${ }^{1}$ Corresponding author: cim24@cornell.edu
}

Key words: viscosity, micellar casein concentrate, Arrhenius model, flow behavior

\section{INTRODUCTION}

Casein and caseinates are valuable food ingredients because of their nutritive value and physicochemical and functional properties. Due to their water-binding, emulsifying, whipping, foaming, and texturizing properties, casein concentrates are used in a range of commercial applications, including protein fortification of dairy foods, and ingredients for beverages, bakery, and meat products (Mulvihill and Ennis, 2003). Traditionally, caseinates and caseins were prepared by either isoelectric precipitation or rennet coagulation (Fox, 2001). Recently, casein preparations obtained by microfiltration (MF) are receiving increasing interest from the food and dairy industry (Affertsholt, 2009). In this process, casein is separated from serum proteins (SP) based on their difference in molecular size (Brans et al., 2004). Additional diafiltration of the retentate can lead to SP removal from the casein concentrate of up to $95 \%$ (Nelson and Barbano, 2005). In casein concentrates obtained by membrane separation, the casein micelles are closer to their native state than in casein preparations obtained by chemical methods, which is why they are typically called micellar casein concentrates (MCC). Micellar casein concentrates and traditional casein ingredients have different functionality, which opens the field for new possible applications (Nelson and Barbano, 2005).

Rheological properties of MCC are very important and have high relevance for any new food application. Such data are necessary for equipment selection and the design of various unit operations and processing steps such as pumping, mixing, heating, and cooling. Rheological data can also provide valuable information about the mouthfeel of the finished product (Hermansson, 1975).

The objective of this study was to evaluate the steady shear rheological properties of MCC under a range of 
concentration, temperature, and shear conditions and to establish a mathematical model able to accurately predict viscosity as a function of these parameters. Such a model will provide the dairy and food industry with critical rheological data necessary for developing commercial applications of micellar casein preparations.

\section{MATERIALS AND METHODS}

\section{Manufacture of MCC}

Micellar casein concentrates were obtained by membrane separation in the pilot plant at Cornell University (Ithaca, NY) according to the procedure described by Zulewska et al. (2009). Pasteurized skim milk was processed at $50^{\circ} \mathrm{C}$ using a uniform transmembrane pressure (UTP) MF system equipped with ceramic Membralox membranes with a $0.1-\mu \mathrm{m}$ pore size. The MF process was continuous bleed-and-feed at a concentration factor of $3 \times$, which resulted in $65 \%$ SP-reduced MCC. To obtain 95\% SP-reduced MCC, the retentates obtained after the first and second MF stages were diluted with reverse osmosis water at a 2:1 ratio by weight (2 parts water, 1 part retentate). Both the $65 \%$ SP-reduced and $95 \%$ SP-reduced retentates were spray dried using a model 1 Niro Atomizer equipped with an FU11 atomizer rotating at 23,000 rpm (Niro Atomizer Inc., Columbia, MD). The inlet air temperature was $200^{\circ} \mathrm{C}$ and the outlet air temperature was $95^{\circ} \mathrm{C}$. The spraydried powders were stored in the dark at $25^{\circ} \mathrm{C}$ until use. For each type of MCC, the processing was replicated 3 times with different batches of milk.

\section{Chemical Analysis}

Fresh, liquid samples of the 65 and 95\% SP-reduced MCC retentates were analyzed for fat, total nitrogen (TN), NPN, and noncasein nitrogen (NCN) using ether extraction (AOAC International, 2005; method 989.05; 33.2.26); Kjeldahl method 991.20, 33.2.11; Kjeldahl method 991.21, 33.2.12; and Kjeldahl method 998.05, 33.2.64 (AOAC International, 2005), respectively. True protein (TP) was calculated by subtracting NPN from TN and then multiplying by 6.38 , casein content $(\mathrm{CN})$ was calculated by subtracting $\mathrm{NCN}$ from TN and multiplying by 6.38 ; and SP content was calculated by subtracting NPN from NCN and multiplying by 6.38 . The SP removal was estimated by Kjeldahl analysis (TN, NPN, and NCN) of the MF permeates according to Hurt et al. (2010).

After spray drying, powders were reconstituted to $10 \%$ solids and their $\mathrm{pH}$ was measured with an Elec- trolyte 9823 electrode (Mettler-Toledo LLC, Columbus, $\mathrm{OH})$. The TS content of the powders was measured by forced-air oven drying (AOAC International, 2005; method 990.20, 33.2.44).

\section{Sample Preparation}

The protein powders were weighed and slowly added to ultrapure water to obtain suspensions of casein concentrations of $2.5,5.0,7.5,10.0$, and $12.5 \%$ (wt/ $\mathrm{vol}$ ). The suspensions were stirred for $30 \mathrm{~min}$ on a stir plate at moderate speed at $25^{\circ} \mathrm{C}$ and then ultrahigh shear mixed using an Ultra-Turrax model T25 fitted with an S25N-18G dispersing tool (IKA Works Inc., Wilmington, NC) for $5 \mathrm{~min}$ at 21,500 rpm. Following the high-shear dispersion, the suspensions were kept under continuous stirring at low speed at $25^{\circ} \mathrm{C}$ for an additional 90 min to allow for full hydration and for the foam to break. A detailed discussion about how rehydration and sample preparation procedure were established is included in the paper by Beliciu and Moraru (2011).

\section{Rheological Analysis}

The viscosity and flow behavior of the reconstituted 65 and 95\% SP-reduced MCC were determined using large deformation rheological analyses. Steady-shear rate sweeps were conducted using an advanced rheometric expansion system strain-controlled rheometer equipped with a Peltier temperature control system (TA Instruments, New Castle, DE). Two milliliters of MCC sample was carefully loaded onto a parallelplate fixture (diameter: $50 \mathrm{~mm}$, interplate gap: $1 \mathrm{~mm}$ ) at $20^{\circ} \mathrm{C}$, avoiding the formation of air bubbles. To prevent water evaporation during the measurement, a thin mineral oil film was applied around the geometry's circumference. For test temperatures above $50^{\circ} \mathrm{C}$, an isothermal chamber was installed around the fixture. The temperature of the Peltier element was set to the desired value $\left(0,20,40,60\right.$, or $\left.80^{\circ} \mathrm{C}\right)$ and once the test temperature was reached, the samples were allowed a 60-s temperature equilibration and relaxation step before starting the test. Steady-shear rate sweeps were conducted, with shear rates ranging from 1 to $631 \mathrm{~s}^{-1}$ at a frequency of $6.28 \mathrm{rad} / \mathrm{s}(1 \mathrm{~Hz})$ in clockwise and counterclockwise direction. For each sample, triplicate measurements were performed.

The data were collected and analyzed using the Orchestrator software (TA Instruments). The Fit Model component of the software was used to fit the data to rheological models. 


\section{Statistical Analysis}

The software JMP 8.0 (SAS Institute Inc., Cary, NC) was used for statistical analyses of the data. Analysis of variance was used to determine the effect of SP removal, concentration, and temperature. Significant differences among samples were determined by the Tukey honestly significant difference (HSD) test at $P \leq 0.05$.

\section{RESULTS AND DISCUSSION}

\section{Chemical Composition of the Powders}

A summary of the chemical composition of the 65 and 95\% SP-reduced MCC powders is presented in Table 1. The CN in the $65 \%$ SP-reduced MCC was $51.10 \%$, which equates to a casein-to-TP ratio $(\mathrm{CN} \% \mathrm{TP})$ of 90.23\%. For the 95\% SP-reduced MCC, the CN was $80.47 \%$ and the CN\% TP was $95.64 \%$, due to the additional removal of SP and NPN from the retentate during the 2 diafiltration steps in the production process. The diafiltration also led to a significant reduction of lactose to a final concentration of $3.70 \%$ in the $95 \%$ SP-reduced MCC compared with $30.34 \%$ lactose in the $65 \%$ SP-reduced MCC. The fat content was similar in the 2 types of MCC powders. A detailed discussion about the composition of these retentates is included in the paper by Hurt and Barbano (2010).

\section{Flow Behavior}

Figure 1a shows the flow curves of MCC dispersions, with casein concentrations ranging from 2.5 to $12.5 \%$ at $20^{\circ} \mathrm{C}$. The MCC with $2.5 \%$ concentration had virtually a Newtonian behavior, as the viscosity did not change with shear rate. The MCC with casein concentrations above 5\% exhibited mild shear-thinning behavior, whereas MCC with casein concentrations $\geq 7.5 \%$ exhibited clear non-Newtonian shear-thinning behavior across the investigated range of shear rates. The flow behavior was also affected by temperature, with more pronounced shear thinning being observed at low temperatures. Figure $1 \mathrm{~b}$ shows the flow curves for the $12.5 \%$ MCC, which exhibited clear shear-thinning behavior for all of the investigated temperatures $\left(0-80^{\circ} \mathrm{C}\right)$. These observations are in agreement with the study by Beliciu and Moraru (2011), who reported that the rheological behavior of dispersions of MCC, soy proteins, and their mixtures show 2 distinct regimens of flow behavior. In the dilute regimen, at protein concentration $\leq 5 \%$, the samples behaved as Newtonian fluids, whereas at protein concentration $\geq 7.5 \%$, non-Newtonian, shear-thinning behavior was observed. Shear-thinning behavior is typical for many soft foods, including dairy products, and has implications for both processing (i.e., pumping and pipe flow) and the mouthfeel of food products that contain MCC.

The dependence of viscosity on shear rate for concentrated milk products has been described in the literature using various models, including the power law model (Vélez-Ruiz and Barbosa-Cánovas, 1998; Solanki and Rizvi, 2001), the Bingham model (Bienvenue et al., 2003a), and the Herschel-Bulkley model (Vélez-Ruiz and Barbosa-Cánovas, 1998).

The Herschel-Bulkley model has the following mathematical form:

$$
\sigma=\kappa(\dot{\gamma})^{n}+\sigma_{0}
$$

where $\sigma$ is the shear stress $(\mathrm{Pa}), \kappa$ is the consistency coefficient $\left(\mathrm{Pa} \cdot \mathrm{s}^{n}\right), \dot{\gamma}$ is the shear rate $\left(\mathrm{s}^{-1}\right), n$ is the flow behavior index, and $\sigma_{0}$ is the yield stress $(\mathrm{Pa})$. This model is very general and it can be used to describe the flow behavior of Newtonian $\left(\sigma_{0}=0\right.$ and $\left.n=1\right)$, power law $(0<n<1$ for shear thinning and $1<n<\infty$ for shear thickening), and Bingham plastic behavior $(n=$ 1; Steffe, 1996).

The viscosity data for all the MCC samples was fitted to the Herschel-Bulkley model and the values for $\kappa$, $n$, and $\sigma_{0}$ were determined. The fit for all data sets was very good, with the coefficient of determination $>98 \%$ in all cases. For all of the MCC samples, the yield stress was very small $\left(\sigma_{0}<0.04 \mathrm{~Pa}\right)$ for the casein concentrations and temperatures evaluated (data not shown).

Flow indices $(n)$ for both the $95 \%$ SP-reduced and $65 \%$ SP-reduced MCC dispersions are presented in Table 2 . In Table 2 , statistically significant $(P \leq 0.05)$

Table 1. Composition of 65 and 95\% serum protein- (SP) reduced micellar casein concentrates (MCC), on dry solids basis (\% by weight)

\begin{tabular}{lcccccccccccc}
\hline & \multicolumn{10}{c}{ Composition $^{1}$} \\
\cline { 2 - 12 } Item & TN & NPN & NCN & TP & CN & CN\% TP & SP & MC & Fat & Lactose & pH \\
\hline $65 \%$ SP-reduced MCC & 57.92 & 1.29 & 6.82 & 56.63 & 51.10 & 0.90 & 5.53 & 3.33 & 2.22 & 30.34 & 6.91 \\
$95 \%$ SP-reduced MCC & 84.56 & 0.42 & 4.09 & 84.14 & 80.47 & 0.96 & 3.67 & 4.55 & 2.74 & 3.70 & 7.20 \\
\hline
\end{tabular}

${ }^{1} \mathrm{TN}=$ total nitrogen $\times 6.38 ; \mathrm{NPN}=\mathrm{NPN} \times 6.38 ; \mathrm{NCN}=$ noncasein nitrogen $\times 6.38 ; \mathrm{TP}=$ true protein $(\mathrm{TN}-\mathrm{NPN}) ; \mathrm{CN}=$ casein content $(\mathrm{TN}-\mathrm{NCN}) ; \mathrm{CN} \% \mathrm{TP}=$ casein as percentage of true protein; $\mathrm{SP}=\mathrm{TP}-\mathrm{CN} ; \mathrm{MC}=$ moisture content. Values are averages of 3 experimental replicates. 



Figure 1. Apparent viscosity as a function of shear rate for $95 \%$ serum protein- (SP) reduced micellar casein concentrate (MCC) dispersions of (a) 2.5 to $12.5 \%$ casein concentration at $20^{\circ} \mathrm{C}$ and (b) $12.5 \%$ casein concentration at temperatures between 0 and $80^{\circ} \mathrm{C}$. Plotted are mean values $(\mathrm{n}=3) \pm 1 \mathrm{SD}$.

differences in $n$ values among samples of varying casein concentrations are indicated by different letters. The shear-thinning behavior of the samples is reflected by $n<1$.

It has been shown before that the flow behavior and the extent of shear thinning of milk concentrates
(Stepp and Smith, 1991; Vélez-Ruiz and Barbosa-Cánovas, 1998; Solanki and Rizvi, 2001; Bienvenue et al., 2003a,b) and MF retentates (Solanki and Rizvi, 2001) depend on factors such as composition, concentration, temperature, and storage time. In this work, the shearthinning behavior of the MCC was more prevalent (i.e., the flow index $n$ decreased) with increasing concentration and decreasing temperature. Samples of casein concentrations of 2.5 and $5.0 \%$ had $n$ very close to 1 , which confirms that these MCC dispersions have a virtually Newtonian behavior, whereas samples of higher concentrations had $n<1$. The $65 \% \mathrm{SP}$-reduced and the 95\% SP-reduced MCC showed very similar flow behavior and $n$ across the concentration and temperature ranges used in this study (see Table 2).

\section{Effect of SP Removal on the Viscosity of MCC}

Because many of the MCC dispersions behaved as non-Newtonian fluids across the investigated range of shear rates, with the viscosity changing as a function of the shear rate, the term apparent viscosity $\left(\boldsymbol{\eta}_{\text {app }}\right)$ will be used to describe their resistance to flow. To evaluate the effect of composition and temperature on the viscosity of the MCC, comparisons were made using the $\eta_{\text {app }}$ at a shear rate of $100 \mathrm{~s}^{-1}\left(\boldsymbol{\eta}_{\mathbf{1 0 0}}\right)$. This shear rate was selected due to its association with stirring, pumping, pipe flow, other processing operations, as well as mastication (Steffe, 1996).

The effect of SP removal on viscosity was evaluated by comparing the $\eta_{\text {app }}$ data for the $65 \%$ SP-reduced MCC and the $95 \%$ SP-reduced MCC. It is important to note that, at the same casein concentration, the $65 \%$ SP-removed MCC contained more SP, more lactose, and more NPN, and consequently higher TS than the $95 \%$ SP-removed MCC (Table 1). As seen in Figures $2 \mathrm{a}$ and $2 \mathrm{~b}$, at the same casein concentration and temperature, the $65 \%$ SP-reduced MCC dispersions exhibited lower values of $\eta_{100}$ than the $95 \%$ SP-reduced MCC $(P \leq$ 0.05 ) with the exception of $80^{\circ} \mathrm{C}$ (Figure $2 \mathrm{a}$ ) and $2.5 \%$ (Figure 2b). The almost 10-fold higher lactose content in the $65 \%$ SP-reduced MCC as compared with the $95 \%$ SP-reduced MCC would be expected to increase viscosity. In the study by Morison and Mackay, (2001), the addition of varying amounts of lactose to whey protein concentrates significantly increased their viscosity. The higher viscosity of the higher-purity MCC is consistent with previous reports (Solanki and Rizvi, 2001) and indicates that casein is the main contributor to the viscosity of the MCC. This means that the soluble components (SP, lactose, NPN, and minerals) interfered with the casein-casein interactions and, thus, reduced the viscosity of the $65 \%$ SP-reduced MCC samples. 
Table 2. Flow indices for 65 and $95 \%$ serum protein- $(\mathrm{SP})$ reduced micellar casein concentrates $[\mathrm{MCC} ;$ mean values $(\mathrm{n}=3) \pm 1 \mathrm{SD}]$

\begin{tabular}{|c|c|c|c|c|c|c|}
\hline MCC type & $\begin{array}{c}\text { Concentration } \\
(\%)\end{array}$ & \multicolumn{5}{|c|}{ Flow index $n$} \\
\hline \multirow[t]{4}{*}{$95 \%$ SP-reduced MCC } & 2.5 & $0.97 \pm 0.02^{\mathrm{a}}$ & $0.99 \pm 0.00^{\mathrm{a}}$ & $0.99 \pm 0.00^{\mathrm{a}}$ & $0.98 \pm 0.00^{\mathrm{a}}$ & $0.98 \pm 0.00^{\mathrm{a}}$ \\
\hline & 7.5 & $0.88 \pm 0.01^{\mathrm{a}}$ & $0.95 \pm 0.02^{\mathrm{a}}$ & $0.99 \pm 0.01^{\mathrm{a}}$ & $0.99 \pm 0.00^{\mathrm{a}}$ & $0.99 \pm 0.00^{\mathrm{ab}}$ \\
\hline & 10.0 & $0.75 \pm 0.02^{\mathrm{b}}$ & $0.85 \pm 0.03^{\mathrm{b}}$ & $0.97 \pm 0.01^{\mathrm{a}}$ & $0.99 \pm 0.00^{\mathrm{a}}$ & $0.99 \pm 0.00^{\mathrm{b}}$ \\
\hline & 12.5 & $0.62 \pm 0.07^{\mathrm{c}}$ & $0.71 \pm 0.03^{\mathrm{c}}$ & $0.86 \pm 0.03^{\mathrm{b}}$ & $0.95 \pm 0.01^{\mathrm{b}}$ & $0.98 \pm 0.01^{\mathrm{ab}}$ \\
\hline \multirow{3}{*}{$65 \%$ SP-reduced MCC } & 7.5 & $0.90 \pm 0.08^{\mathrm{a}}$ & $0.97 \pm 0.01^{\mathrm{a}}$ & $0.99 \pm 0.00^{\mathrm{a}}$ & $0.99 \pm 0.00^{\mathrm{a}}$ & $0.99 \pm 0.00^{\mathrm{ab}}$ \\
\hline & 10.0 & $0.72 \pm 0.03^{\mathrm{b}}$ & $0.86 \pm 0.03^{\mathrm{b}}$ & $0.97 \pm 0.00^{\mathrm{a}}$ & $0.99 \pm 0.00^{\mathrm{a}}$ & $0.99 \pm 0.00^{\mathrm{b}}$ \\
\hline & 12.5 & $0.57 \pm 0.08^{\mathrm{c}}$ & $0.64 \pm 0.03^{\mathrm{c}}$ & $0.81 \pm 0.03^{\mathrm{b}}$ & $0.95 \pm 0.02^{\mathrm{b}}$ & $0.99 \pm 0.01^{\mathrm{ab}}$ \\
\hline
\end{tabular}

${ }^{\mathrm{a}-\mathrm{c}}$ Means within a column not sharing a common superscript are statistically different $(P<0.05)$.

\section{Effect of Temperature on the Viscosity of MCC}

The $\eta_{\text {app }}$ at $100 \mathrm{~s}^{-1}\left(\eta_{100}\right)$ versus temperature for the 95\% SP-reduced MCC is plotted in Figure 3, in which the solid points represent the experimentally measured values. Temperature had a significant effect $(P \leq 0.05)$ on the $\eta_{\text {app }}$ of MCC, with $\eta_{100}$ decreasing with increasing temperature, at all casein concentrations.

The effect of temperature on viscosity of fluids, including concentrated milk products, is commonly described using an Arrhenius-type relationship (Fichtali et al., 1993; Vélez-Ruiz and Barbosa-Cánovas, 1998). In this study, the effect of temperature on the $\eta_{\text {app }}$ of the MCC also followed an Arrhenius relationship:

$$
\eta_{100}=A e^{\left(\frac{E_{a}}{R T}\right)},
$$

where $\eta_{100}$ is the $\eta_{\text {app }}$ at shear rate $100 \mathrm{~s}^{-1}(\mathrm{~Pa} \cdot \mathrm{s}), A$ is a preexponential constant $(\mathrm{Pa} \cdot \mathrm{s}), E_{a}$ is the activation energy of flow $(\mathrm{J} / \mathrm{mol}), R$ is the universal gas constant $[8.314 \mathrm{~J} /(\mathrm{K} \cdot \mathrm{mol})]$, and $T$ is the absolute temperature $(\mathrm{K})$.

The $E_{a}$ and $A$ were determined by linear regression analysis of $1 / T$ versus $\log (\eta)$ data for each concentration and replicate. The mean values from the 3 replicates of $A$ and $E_{a}$ were used to calculate the $\eta_{\text {app }}$ of 95\% SP-reduced MCC of different concentrations as a function of temperature, and were plotted as lines in Figure 3. As observed in this graphic representation, the calculated values were very close to the experimental values. A similar analysis was conducted for the $65 \%$ SP-reduced MCC.

The activation energy indicates the sensitivity of the viscosity to a change in temperature, with higher
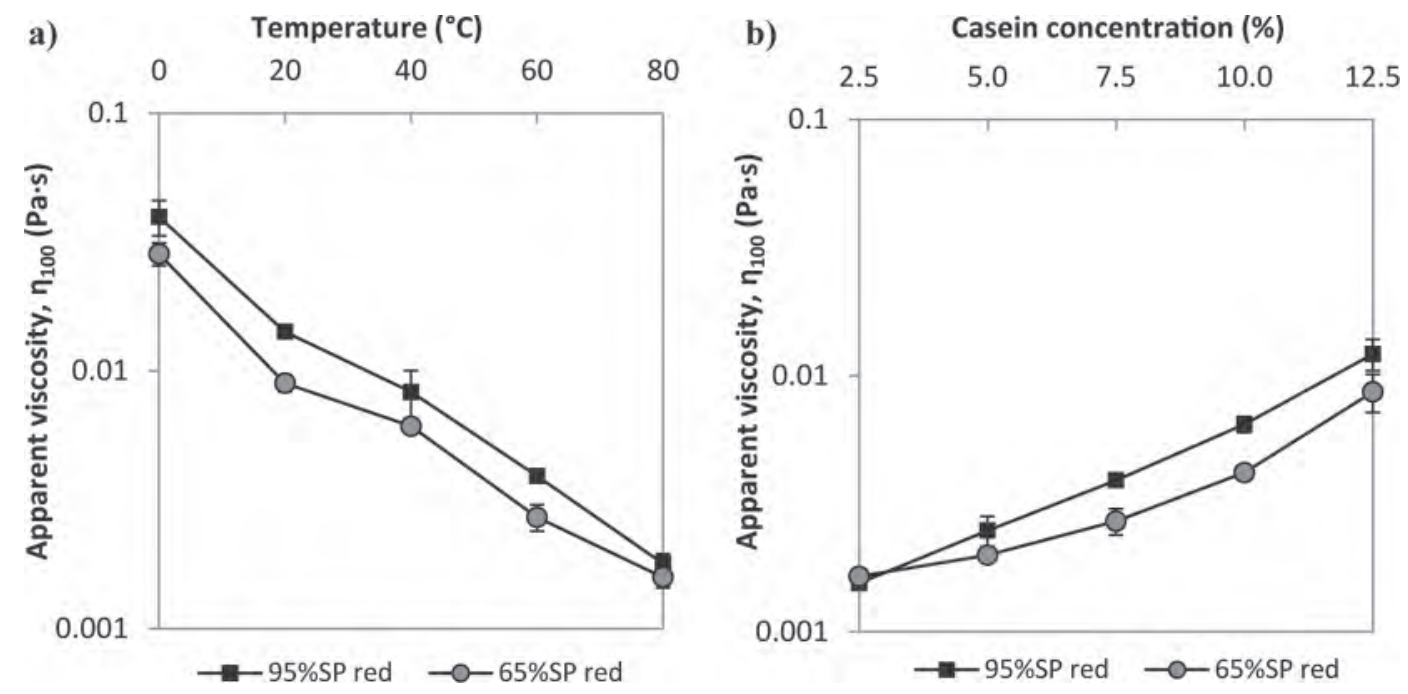

Figure 2. Comparison of the viscosity of $65 \%$ serum protein- (SP) reduced (red) and $95 \%$ SP-reduced micellar casein concentrates (MCC): (a) $7.5 \%$ casein concentration in the temperature range 0 to $80^{\circ} \mathrm{C}$; (b) range of casein concentrations from 2.5 to $12.5 \%$ at $60^{\circ} \mathrm{C}$. Plotted are mean values $(\mathrm{n}=3) \pm 1 \mathrm{SD}$. 


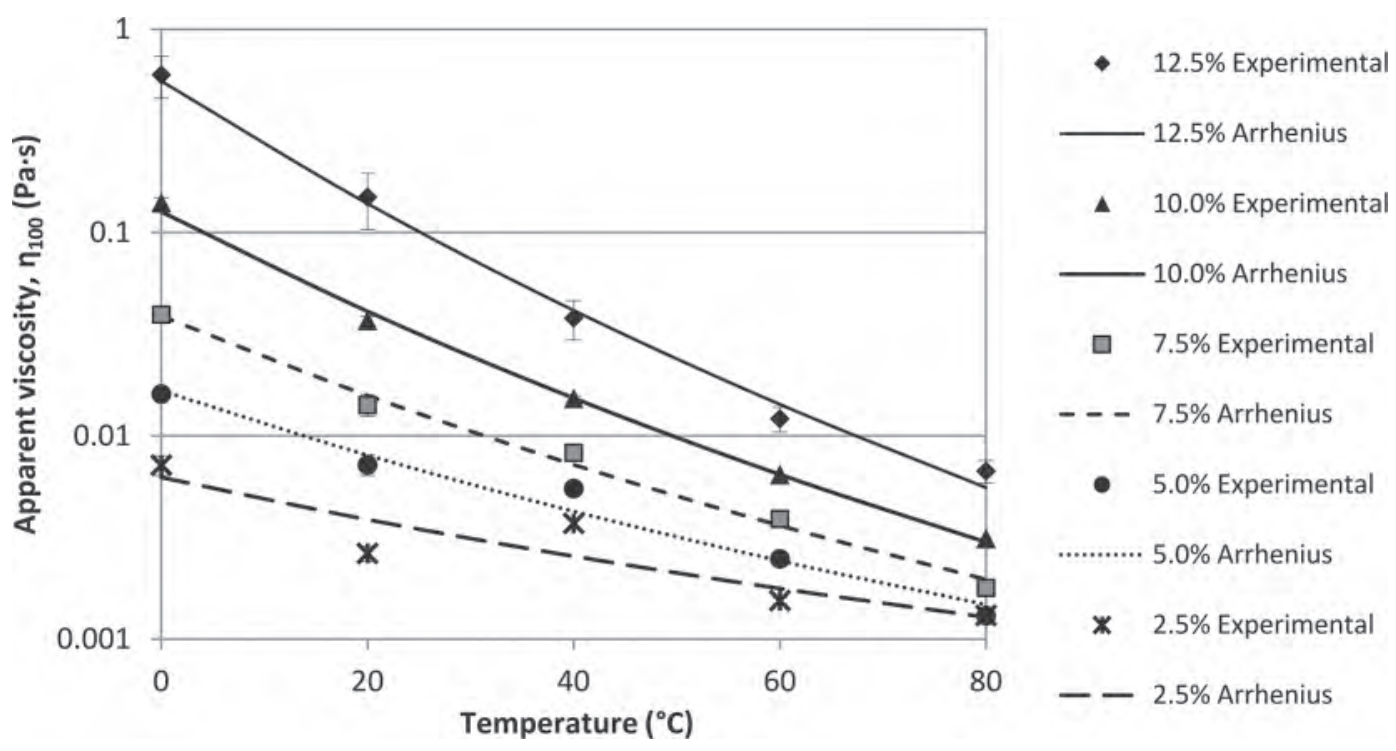

Figure 3. Comparison of experimental apparent viscosity $\left(\eta_{100}\right)$ values (points) with values calculated using an Arrhenius relationship (lines), for $95 \%$ serum protein- (SP) reduced micellar casein concentrates $(\mathrm{MCC})$ as a function of temperature in the range of 0 to $80^{\circ} \mathrm{C}$. Plotted are mean values $(\mathrm{n}=3) \pm 1 \mathrm{SD}$.

values indicating that the viscosity is more sensitive to temperature changes (Steffe, 1996). The values of $E_{a}$ for the 65 and $95 \%$ SP-reduced MCC were in the ranges of 15.1 to 49.9 and 15.8 to $46.2 \mathrm{~kJ} / \mathrm{mol}$, respectively, and were comparable with values reported in the literature for different types of skim milk concentrates (Solanki and Rizvi, 2001). The $E_{a}$ was found to increase linearly with increasing casein concentration of the 95\% SPreduced MCC dispersions $\left(\mathrm{R}^{2}>99 \%\right.$; Figure $\left.4 \mathrm{a}\right)$ and $65 \%$ SP-reduced MCC dispersions (data not shown). This is in agreement with previous reports that found a linear correlation between activation energy $E_{a}$ and TS content (Chang and Hartel, 1997; Solanki and Rizvi, 2001), although other authors reported a nonlinear dependence of $E_{a}$ with concentration and storage time (Vélez-Ruiz and Barbosa-Cánovas, 1998).

The preexponential parameter $A$ indicates a fluid's internal resistance to flow that is free from the influence of temperature (Giap, 2010). The values of $A$ for the 65 and $95 \%$ SP-reduced MCC were in the ranges of $7.71 \times 10^{-6}$ to $1.72 \times 10^{-10}$ and $6.26 \times 10^{-6}$ to $1.23 \times$ $10^{-9} \mathrm{~Pa} \cdot \mathrm{s}$, respectively. The values of the preexponen-

b)

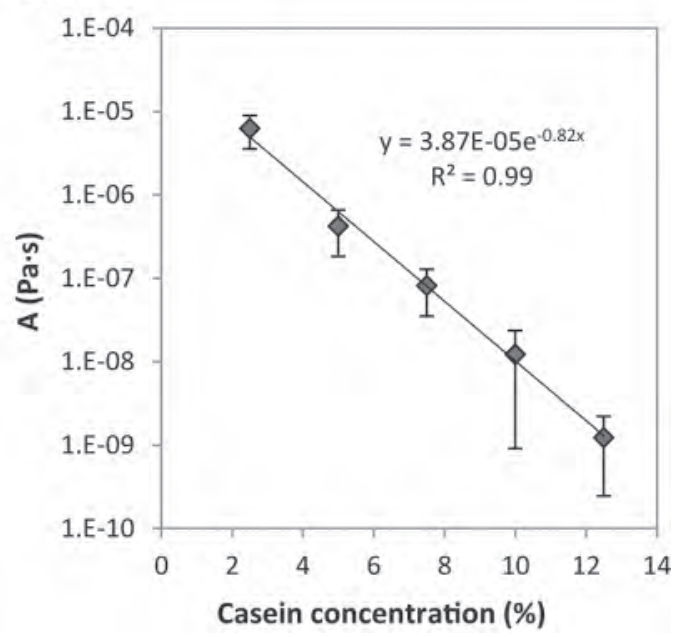

Figure 4. Arrhenius constants: (a) activation energy $\mathrm{E}_{\mathrm{a}}$; (b) preexponential parameter $\mathrm{A}$ as function of casein concentration for $95 \%$ serum protein- (SP) reduced micellar casein concentrates (MCC). Plotted are mean values $(\mathrm{n}=3) \pm 1 \mathrm{SD}$. 




Figure 5. Comparison of experimental apparent viscosity $\left(\eta_{100}\right)$ values (points) with values calculated using an exponential relationship (lines) for $95 \%$ serum protein- (SP) reduced micellar casein concentrates (MCC) as a function of casein concentration in the range of 2.5 to $12.5 \%$. Plotted are mean values $(\mathrm{n}=3) \pm 1 \mathrm{SD}$.

tial parameter $A$ decreased exponentially with casein concentration for both 95\%-SP reduced MCC (Figure $4 \mathrm{~b}$ ) and $65 \%$-SP reduced MCC (data not shown).

\section{Effect of Concentration on the Viscosity of MCC}

The $\eta_{100}$ of 65 and $95 \%$ SP-reduced MCC increased with increasing casein concentration, at all temperatures (see points in Figure 5 for the 95\% SP-reduced MCC). This increase in viscosity was more pronounced with increasing concentration; as the concentration of TS (particularly casein) increased, the distance between the casein micelles decreased, leading to a subsequent increase in electrostatic repulsion. As 2 particles with the same charge are trying to avoid each other, they alter their flow path, thus increasing the resistance to flow of the liquid in which they are suspended (İbanoğlu, 2002).

The effect of concentration on viscosity has frequently been described as either a power law (Towler, 1974; Vitali and Rao, 1982) or as an exponential relationship (Rao et al., 1984; Beliciu and Moraru, 2011). In the current study, for all MCC, the increase in viscosity with concentration followed an exponential relationship, at all tested temperatures:

$$
\eta_{100}=a_{1} e^{a_{2} C}
$$

where $a_{1}$ and $a_{2}$ are constants and $C$ is the casein concentration (\%).

The constants $a_{1}$ and $a_{2}$ were determined from linear regression analysis of $C$ versus $\log (\eta)$ data for all MCC. The values for these coefficients were in agreement with values reported previously by Beliciu and Moraru (2011).

To verify the goodness of the model, the calculated coefficients were then used to calculate the $\eta_{\text {app }}$ of the $65 \%$ and $95 \%$ SP-reduced MCC at different temperatures for the concentration range $2.5-12.5 \%$, and the calculated values are plotted as lines in Figure 5, where it can be noticed that the calculated values and experimentally measured values are very close. This was the case for both MCC types (data for the 65\% SP-reduced MCC is not shown).

\section{Development of an Overall Predictive Model for MCC Viscosity}

For many practical applications, it is very useful to have a mathematical model able to accurately predict viscosity at a given shear rate, temperature, and concentration. The determined dependencies of $\eta_{100}$ on temperature and concentration of MCC dispersions were used to develop an overall predictive model for both types of MCC. By combining the Arrhenius relationship for the temperature-viscosity dependence and 
a)

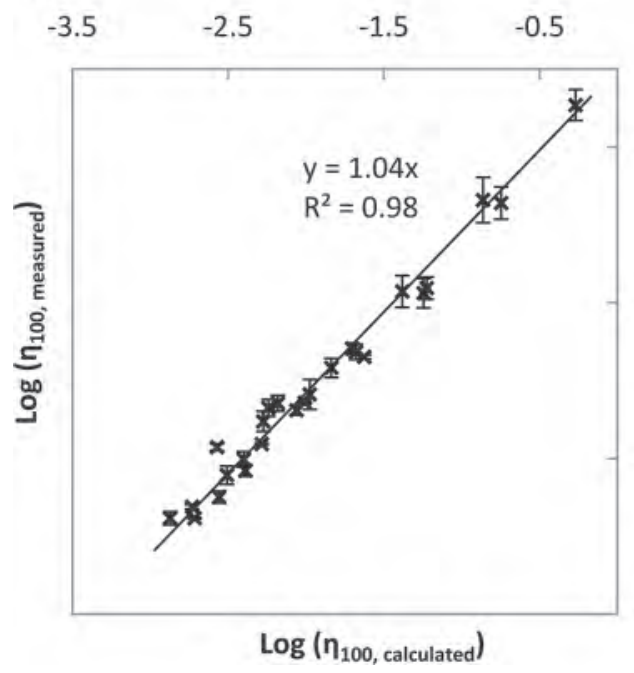

b)

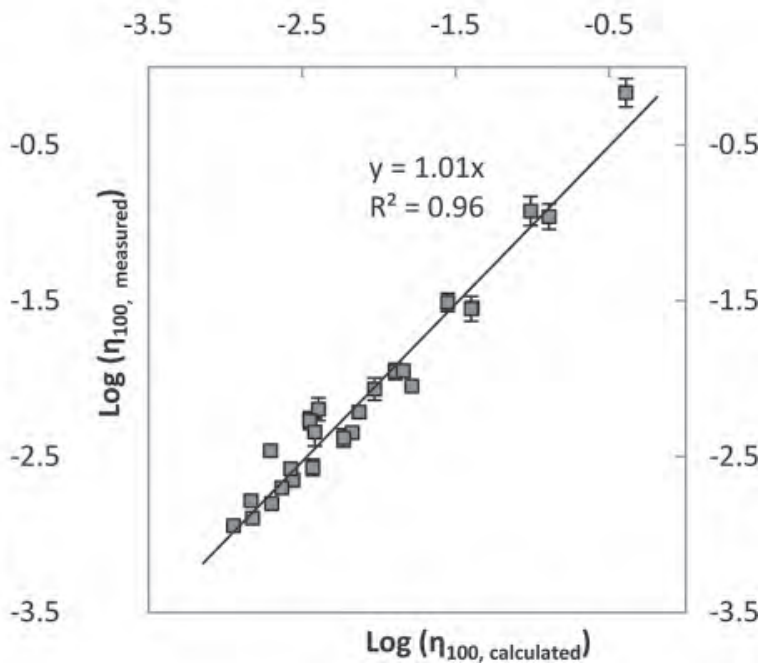

Figure 6. Model fit: measured versus calculated logarithm of apparent viscosity $\left[\log \left(\eta_{100}\right)\right]$ values for (a) $95 \%$ serum protein- $(\mathrm{SP})$ reduced micellar casein concentrates (MCC) and (b) $65 \%$ SP-reduced MCC. Plotted are mean values $(\mathrm{n}=3) \pm 1$ SD.

the exponential relationship between concentration and viscosity, the following modified Arrhenius model was developed:

$$
\eta_{100}=a_{1} e^{\left(a_{2} C+\frac{a_{3}+a_{4} C}{R T}\right)},
$$

where $\eta_{100}$ is the $\eta_{\text {app }}$ at the shear rate $100 \mathrm{~s}^{-1}(\mathrm{~Pa} \cdot \mathrm{s}) ; a_{1}$ $(\mathrm{Pa} \cdot \mathrm{s}), a_{2}$ (dimensionless), $a_{3}(\mathrm{~J} / \mathrm{mol})$, and $a_{4}(\mathrm{~J} / \mathrm{mol})$ are constants; $C$ is the casein concentration (\%), $R$ is the universal gas constant $[8.314 \mathrm{~J} /(\mathrm{K} \cdot \mathrm{mol})]$, and $T$ is the absolute temperature $(\mathrm{K})$. The calculated constants $a_{1}, a_{2}, a_{3}$, and $a_{4}$ for the 65 and $95 \%$ SP-reduced MCC dispersions are shown in Table 3.

To evaluate the fit of the developed model, a regression analysis of the measured versus predicted values of $\eta_{100}$ was performed, both for the $95 \%$ SP-reduced and for the $65 \%$ SP-reduced MCC. The model was found to predict $\eta_{\text {app }}$ very well in the range of tested concentrations and temperatures, with a coefficient of determination of $98 \%$ for the $95 \%$ SP-reduced MCC (Figure 6a) and a coefficient of determination of $96 \%$ for the 65\% SP-reduced MCC (Figure 6b). As slight differences in viscosity can occur due to minor fluctuations in composition of testing conditions, from a practical perspective, it is more useful to predict the order of magnitude of viscosity than the actual values of viscosity. For this reason, the fit of the model was evaluated by plotting the logarithm of viscosity instead of the actual viscosity values in Figure 6. Nonetheless, the regression parameters were almost the same when comparing the actual viscosity values (data not shown).

Furthermore, the model was validated by generating a set of experimental viscosity data that was not used in the development of the model. For the $95 \%$ SP-reduced MCC, the validation was conducted for the following samples: $6 \%$ casein concentration at $30^{\circ} \mathrm{C}$ and $70^{\circ} \mathrm{C} ; 9 \%$ casein concentration at $0^{\circ} \mathrm{C}$; and $13 \%$ casein concentration at 0,10 , and $50^{\circ} \mathrm{C}$. For the $65 \% \mathrm{SP}$-reduced MCC, the validation was conducted for $4 \%$ casein concentration at 30 and $70^{\circ} \mathrm{C}$, and $11 \%$ casein concentration at 10 and $30^{\circ} \mathrm{C}$. The predicted versus measured $\eta$ values are shown in Figure 7 . The slope of the regression line of $\log \left(\eta_{100, \text { measured }}\right)$ versus $\log \left(\eta_{100, \text { predicted }}\right)$ is basically 1 and the correlation coefficient is very close to 1 for both types of MCC, which demonstrates the strength of the

Table 3. Determined numerical constants for the predictive model (see Equations 4 and 8 ) for the 65 and $95 \%$ serum protein- (SP) reduced micellar casein concentrates [MCC; mean values $(\mathrm{n}=3) \pm 1 \mathrm{SD}$ ]

Numerical constant




a)

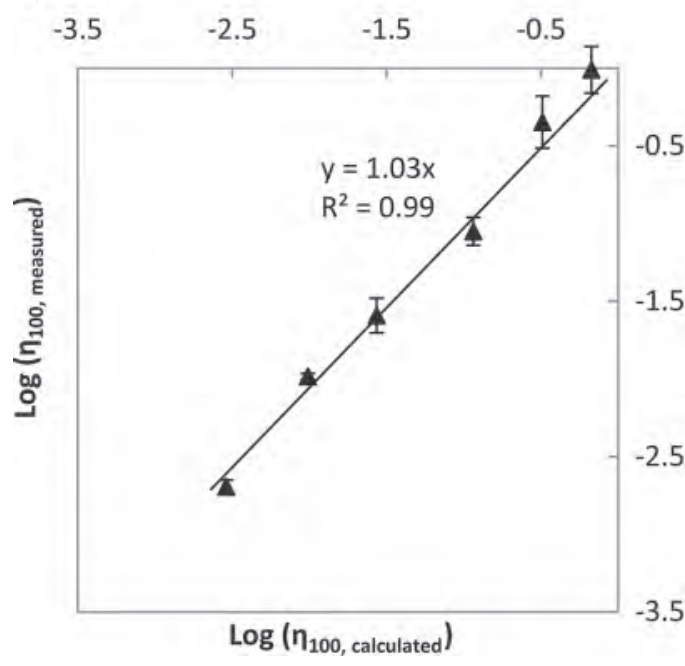

b)



Figure 7. Model validation: measured versus calculated logarithm of apparent viscosity $\left[\log \left(\eta_{100}\right)\right]$ values for (a) $95 \%$ serum protein- $(\mathrm{SP})$ reduced micellar casein concentrates (MCC) and (b) 65\% SP-reduced MCC. Plotted are mean values $(\mathrm{n}=3) \pm 1$ SD.

model in predicting the $\eta_{\text {app }}$ of MCC dispersions for a wide range of shear rates, casein concentrations, and temperatures.

Although $\eta_{100}$ is the most relevant for a range of practical applications, it may be useful for certain situations to be able to predict viscosity at other shear rates. This can be easily done for each temperature, concentration and MCC type by using the predicted values for $\eta_{100}$ and the value of the flow index $n$.

Because the values for yield stress were very small, as a simplifying assumption it can be considered that the MCC have a power law behavior. After considering $\sigma_{0}=0$ in Equation 1, the equation for the $\eta_{\text {app }}$ can be rewritten as

$$
\eta_{\dot{\gamma}}=\kappa(\dot{\gamma})^{n-1}
$$

where $\eta_{\dot{\gamma}}$ is the $\eta_{\text {app }}$ at any shear rate $(\dot{\gamma})>0, n$ is the flow behavior index, and $\kappa$ is the consistency coefficient $\left(\mathrm{Pa} \cdot \mathrm{s}^{n}\right)$.

$$
\text { At } \dot{\gamma}=100 \mathrm{~s}^{-1} \text {, Equation } 5 \text { will become }
$$

$$
\eta_{100}=\kappa(100)^{n-1} .
$$

By combining Equations 5 and 6, the following equation will be obtained:

$$
\eta_{\dot{\gamma}}=\eta_{100}\left(\frac{\dot{\gamma}}{100}\right)^{n-1}
$$

Further, by substituting Equation 4 in Equation 7, an equation that will allow the prediction of $\eta_{\text {app }}$ at any shear rate $>0$, temperature, and concentration will be obtained:

$$
\eta_{(\dot{\gamma}, T, C)}=a_{1} e^{\left(a_{2} C+\frac{a_{3}+a_{4} C}{R T}\right)}\left(\frac{\dot{\gamma}}{100}\right)^{n-1}
$$

where $\eta_{(\dot{\gamma}, T, C)}$ is the $\eta_{\text {app }}$ at any shear rate $(\dot{\gamma})>0 \mathrm{~s}^{-1}$ for the temperature $(T)$ and concentration $(C)$ ranges investigated in this study.

To test the accuracy of this model, values for $\eta_{\text {app }}$ for the $95 \%$ SP-reduced MCC dispersions of $12.5 \%$ casein concentration in the range of shear rates from 1 to $631 \mathrm{~s}^{-1}$ were predicted using Equation 8 and plotted against experimentally obtained values (Figure 8). The fit for the data across all shear rates and temperatures was very good $\left(R^{2}=99 \%\right)$. The model was able to predict very well the order of magnitude of viscosity, with the slope of the regression line for the measured versus calculated $\log \left[\eta_{(\dot{\gamma}, T, C)}\right]$ being very close to 1 (see example for $12.5 \%$ in Figure 8a). The model resulted in a slight underestimation of the actual viscosity values, though. The most significant underestimation, of over $20 \%$, occurred for the $12.5 \%$ MCC (see example in Figure $8 \mathrm{~b}$ ), whereas for the lower concentrations, the underestimation was smaller than $10 \%$ (data not shown).

Nonetheless, as mentioned before, this model is extremely powerful and accurate for estimating the order of magnitude of viscosity, which makes it very useful for 
a)

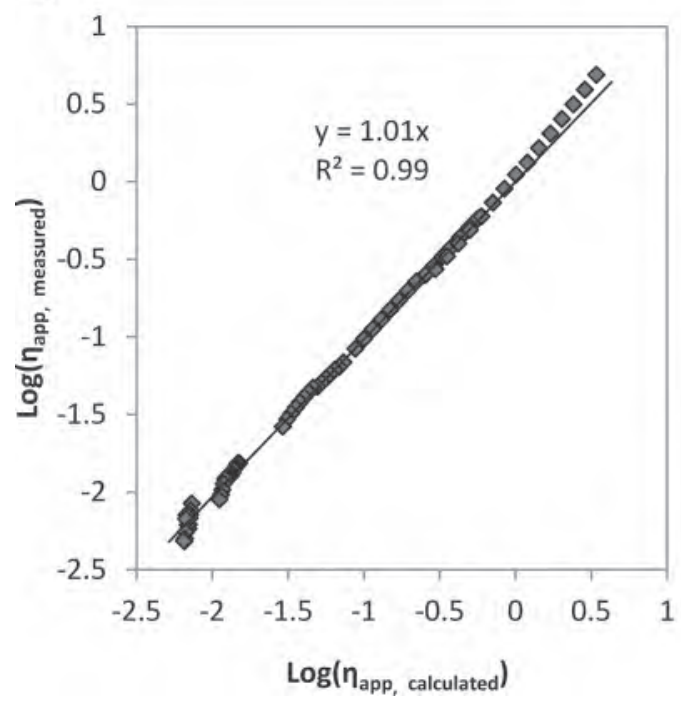

b)

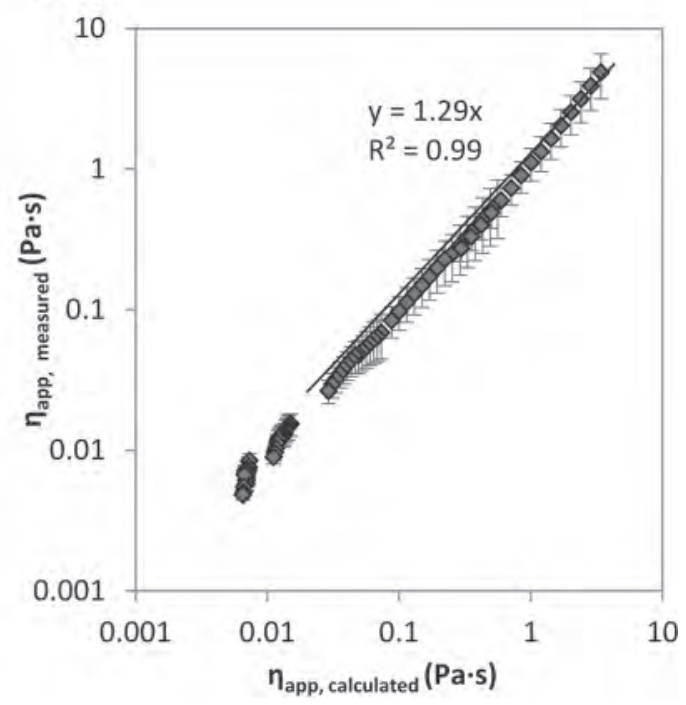

Figure 8. General model fit: measured versus calculated (a) logarithm of apparent viscosity $\left[\log \left(\eta_{\text {app }}\right)\right]$ and (b) $\eta_{\text {app }}$ values for $95 \%$ serum protein- (SP) reduced micellar casein concentrates (MCC) of casein concentration $12.5 \%$, for shear rates between 1 and $631 \mathrm{~s}^{-1}$ and temperatures between 0 and $80^{\circ} \mathrm{C}$. Plotted are mean values $(\mathrm{n}=3) \pm 1 \mathrm{SD}$ (SD for $8 \mathrm{~b}$ only).

designing application of the types of MCC evaluated in this study.

\section{CONCLUSIONS}

The study showed that the $\eta_{\text {app }}$ of MCC increased with concentration and decreased with temperature, and at casein concentrations $\geq 7.5 \%$, the MCC started showing clear shear-thinning behavior. The viscosity of the micellar casein preparations was affected by the SP removal, with higher SP reduction leading to higher viscosity in dispersions with the same casein concentrations. A modified Arrhenius model that incorporates the temperature and concentration dependencies was developed, and was able to predict very accurately MCC $\eta_{\text {app }}$ for a wide range of shear rates, temperatures, and concentrations, of relevance for the practical use of these casein ingredients. The rheological data generated in this study and the predictive model developed will provide the dairy and food industry with critical data necessary for developing applications of micellar casein preparations.

\section{ACKNOWLEDGMENTS}

The authors thank the New York State Milk Promotion Advisory Board for funding this research, and acknowledge the support of the Summer Scholars Program at Cornell University (Ithaca, NY) for author Doehner. We also thank D. M. Barbano, J. Zulewska, Mark Newbold, Emily Hurt, Michael Adams, Chassidy
Coon (all of the Department of Food Science, Cornell University), and the Dairy Plant staff from the Department of Food Science at Cornell University for help with processing and chemical analyses of the casein concentrates.

\section{REFERENCES}

Affertsholt, T. 2009. The market for dairy ingredients based on separation technology. 3A Business Consulting Report. 3A Business Consulting, Aarhus C, Denmark.

AOAC International. 2005. Official methods of analysis of AOAC International. 18th ed. AOAC International, Gaithersburg, MD.

Beliciu, C. M., and C. I. Moraru. 2011. The effect of protein concentration and heat treatment temperature on micellar casein-soy protein mixtures. Food Hydrocoll. 25:1448-1460.

Bienvenue, A., R. Jiménez-Flores, and H. Singh. 2003a. Rheological properties of concentrated skim milk: Importance of soluble minerals in the changes in viscosity during storage. J. Dairy Sci. $86: 3813-3821$.

Bienvenue, A., R. Jimenez-Flores, and H. Singh. 2003b. Rheological properties of concentrated skim milk: Influence of heat treatment and genetic variants on the changes in viscosity during storage. J. Agric. Food Chem. 51:6488-6494.

Brans, G., C. G. P. H. Schroën, R. G. M. van der Sman, and R. M. Boom. 2004. Membrane fractionation of milk: State of the art and challenges. J. Membr. Sci. 243:263-272.

Chang, Y.-H., and R. W. Hartel. 1997. Flow properties of freeze-concentrated skim milk. J. Food Eng. 31:375-386.

Fichtali, J., F. R. van de Voort, and G. J. Doyon. 1993. A rheological model for sodium caseinate. J. Food Eng. 19:203-211.

Fox, P. F. 2001. Milk proteins as food ingredients. Int. J. Dairy Technol. 54:41-55.

Giap, S. G. E. 2010. The hidden property of Arrhenius-type relationship: Viscosity as a function of temperature. J. Physiol. Sci. 21:29-39.

Hermansson, A.-M. 1975. Functional properties of proteins for foodsFlow properties. J. Texture Stud. 5:425-439. 
Hurt, E., and D. M. Barbano. 2010. Processing factors that influence casein and serum protein separation by microfiltration. J. Dairy Sci. 93:4928-4941.

Hurt, E., J. Zulewska, M. Newbold, and D. M. Barbano. 2010. Micellar casein concentrate production with a $3 \times, 3$-stage, uniform transmembrane pressure ceramic membrane process at $50^{\circ} \mathrm{C}$. J. Dairy Sci. 93:5588-5600.

İbanoğlu, E. 2002. Rheological behaviour of whey protein stabilized emulsions in the presence of gum arabic. J. Food Eng. 52:273-277.

Morison, K. R., and F. M. Mackay. 2001. Viscosity of lactose and whey protein solutions. Int. J. Food Properties 4:441-454.

Mulvihill, D. M., and M. P. Ennis. 2003. Functional milk proteins: Production and utilization. Pages 1175-1228 in Advanced Dairy Chemistry: Volume 1: Proteins. 3rd ed. P. F. Fox and P. L. H. McSweeney, ed. Kluwer Academic, New York, NY.

Nelson, B. K., and D. M. Barbano. 2005. A microfiltration process to maximize removal of serum proteins from skim milk before cheese making. J. Dairy Sci. 88:1891-1900.

Rao, M. A., H. J. Cooley, and A. A. Vitali. 1984. Flow properties of concentrated juices at low temperatures. Food Technol. 38:113119
Solanki, G., and S. S. H. Rizvi. 2001. Physico-chemical properties of skim milk retentates from microfiltration. J. Dairy Sci. 84:23812391.

Steffe, J. F. 1996. Rheological Methods in Food Process Engineering. Freeman Press, East Lansing, MI.

Stepp, B. L., and D. E. Smith. 1991. Effect of concentration and temperature on the density and viscosity of skim milk retentates. Milchwissenschaft 46:484-487.

Towler, C. 1974. Rheology of casein solutions. N.Z. J. Dairy Sci. Tech. 9:155-160.

Vélez-Ruiz, J. F., and G. V. Barbosa-Cánovas. 1998. Rheological properties of concentrated milk as a function of concentration, temperature and storage time. J. Food Eng. 35:177-190.

Vitali, A. A., and M. A. Rao. 1982. Flow behavior of guava puree as a function of temperature and concentration. J. Texture Stud. $13: 275-289$

Zulewska, J., M. Newbold, and D. M. Barbano. 2009. Efficiency of serum protein removal from skim milk with ceramic and polymeric membranes at $50^{\circ}$ C. J. Dairy Sci. 92:1361-1377. 